INPLASY

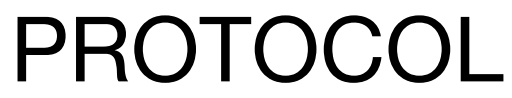

To cite: Wu et al. Can resveratrol protect against renal ischemia/reperfusion injury? A systematic review and meta-analysis of preclinical studies. Inplasy protocol 202140102. doi: 10.37766/inplasy2021.4.0102

Received: 20 April 2021

Published: 20 April 2021

Corresponding author: Mingquan Li

limingquan128@sina.com

Author Affiliation:

Department of Nephrology, Hospital of Chengdu

University of Traditional

Chinese Medicine, Chengdu, Sichuan, P.R. China.

Support: None.

Review Stage at time of this submission: The review has not yet started.

Conflicts of interest:

None declared.

\section{Can resveratrol protect against renal ischemia/reperfusion injury? A systematic review and meta-analysis of preclinical studies}

\author{
Wu, LH1; Wu, L2; Liu, Y3; Jiang, T4; Yang, J5; Li, MQ6.
}

Review question / Objective: We aim to evaluate preclinical evidence and possible mechanism of resveratrol for animal models of renal ischemia/reperfusion (I/R) injury.

Condition being studied: Acute kidney injury (AKI) induced by I/R is a pivotal cause of acute renal failure with high morbidity and mortality. However, there is limited therapy in clinical applications presently. Resveratrol is a natural compound contained in a wide range of plant species, including grapes, peanuts, rhizoma Polygoni cuspidati. Recent studies have shown potential benefits of resveratrol in animal models of renal I/R injury, however, whether these results justify clinical trials is uncertain. Here, we aim to evaluate preclinical evidence and the possible mechanism of resveratrol for animal models of renal I/R injury.

INPLASY registration number: This protocol was registered with the International Platform of Registered Systematic Review and Meta-Analysis Protocols (INPLASY) on 20 April 2021 and was last updated on 20 April 2021 (registration number INPLASY202140102).

\section{INTRODUCTION}

Review question / Objective: We aim to evaluate preclinical evidence and possible mechanism of resveratrol for animal models of renal ischemia/reperfusion (I/R) injury.

Condition being studied: Acute kidney injury (AKI) induced by $I / R$ is a pivotal cause of acute renal failure with high 
morbidity and mortality. However, there is limited therapy in clinical applications presently. Resveratrol is a natural compound contained in a wide range of plant species, including grapes, peanuts, rhizoma Polygoni cuspidati. Recent studies have shown potential benefits of resveratrol in animal models of renal $I / R$ injury, however, whether these results justify clinical trials is uncertain. Here, we aim to evaluate preclinical evidence and the possible mechanism of resveratrol for animal models of renal I/R injury.

\section{METHODS}

Search strategy: The literature search will be conducted by two researchers (Lihua Wu \& Yu Liu) independently in the form of "back-to-back", discrepancies regarding included studies will be discussed and settled by a third reviewer (Mingquan Li). The following term search strategy will be used in the MEDLINE database, and appropriate modifications will be made to suit other databases: "acute kidney injury" [MeSH Terms], "acute renal injury", "kidney ischemia/reperfusion injury ", " renal ischemia/reperfusion injury", " renal ischemic injury", " resveratrol ". "animals not humans".

Participant or population: Animal models of renal I/R injury.

Intervention: Intervention includes in vivo administration of resveratrol to animals. Drug formulation, dosage, mode of administration, and time interval of administration will not be limited.

Comparator: The control group consisted of a placebo, which may be physiological saline or the related vehicle comprised placebo (e.g. saline or similar vehicle control), or no treatment.

Study designs to be included: In vivo studies.

Eligibility criteria: 1. Types of animals: animal models of renal I/R injury 2 . Intervention include in vivo administration of resveratrol to animals 3 . The control group consisted of a placebo, which may be physiological saline or the related vehicle comprised placebo (e.g. saline or similar vehicle control), or no treatment. 4. Types of outcome evaluated: the primary outcomes were serum creatinine (Scr), blood urea nitrogen (BUN) and pathological changes of renal tissue. Scr and BUN must have one item for a more comprehensive evaluation of kidney function. The second outcome measures were mechanisms of resveratrol for renal I/R injury.

Information sources: We will comprehensively search the following databases: MEDLINE, EMBASE, Cochrane Library, BIOSI Previews database. Additional trials will be searched by reviewing the reference lists of the retrieved articles, conference proceedings, and gray literature. We will contact the original investigators for more complete details of the study to solve questions about eligibility if necessary.

Main outcome(s): Serum creatinine (Scr), blood urea nitrogen (BUN), and pathological changes of renal tissue. Scr and BUN must have one item for a more comprehensive evaluation of kidney function.

Additional outcome(s): The second outcome measures were mechanisms of resveratrol for renal I/R injury.

Quality assessment / Risk of bias analysis: To assess the risk of bias in the included studies, the Systematic Review Center for Laboratory animal Experimentation (SYRCLE) risk of bias tool 10-item scale will be used, which is specifically designed for animal studies. Each item is as follows: (1) sequence generation, (2) baseline characteristics, (3) allocation concealment, (4) random housing, (5) blinding (for trial caregivers and researchers), (6) random outcome assessment, (7) blinding (for outcome assessors), (8) incomplete outcome data, (9) selective outcome reporting and (10) other sources of bias. Each item is assigned a value of 1 point (total score of 10 points). The "yes" judgment indicates that the risk of bias is 
low, and the "no" judgment indicates a high degree of bias risk. If the details of the report are insufficient to assess the appropriate risk of bias, the judgment will be "unclear". For unclear items in the study, contact the corresponding author for details. The quality assessment will be conducted independently by two researchers (Ting Jiang \& Ling Wu). Any disagreement will be resolved through discussion with a third reviewer (Mingquan Li).

Strategy of data synthesis: Continuous outcomes will be presented as a standardized mean difference (SMD) with a 95\% confidence interval (CI) between groups. Binary outcomes will be summarized using odds ratios (ORs) or relative risks (RRs) with a $95 \% \mathrm{Cl}$ for relative effect. The random-effects model will be used to calculate the pooled effect. This model took into account the accuracy of individual studies and the differences between studies and weights of each study. Statistical heterogeneity between trials will be analyzed by $Q$ statistic and 12 test. If quantitative synthesis is not appropriate, qualitative analysis will be carried out. Statistical analyses were performed with STATA version 16.0 (STATA Corporation, College Station, TX, USA), with $P$ values $\leq 0.05$ considered statistically significant. The quality of evidence and strength of recommendations will be assessed by the Grading of Recommendations, Assessment, Development, and Evaluations (GRADE) method.

Subgroup analysis: Subgroup analysis will be further stratified by species, reperfusion duration, route of administration, or timing regimen of a pretreatment when there are sufficient data. If there is significant heterogeneity in the study $(12>50 \%)$ and the four preset subgroups fail to find the source of heterogeneity, then further metaregression analysis will be performed. Meta-regression analysis will be used to study $\geq 10$ primary outcomes. $P<0.05$ was considered statistically significant.
Sensitivity analysis: A sensitivity analysis will be performed on the primary outcomes regardless of whether the preset subgroup analysis could find heterogeneity. Sensitivity analysis will be conducted by removing one study in turn to estimate the influence of each study.

Language: No language restrictions.

Country(ies) involved: China.

Keywords: Resveratrol, renal ischemia/ reperfusion injury, animal renal $\mathbf{I} R$ injury models, systematic review.

Contributions of each author:

Author 1 - Lihua Wu.

Author 2 - Ling Wu.

Author 3 - Yu Liu.

Author 4 - Ting Jiang.

Author 5 - Ju Yang.

Author 6 - Mingquan Li. 